\title{
Fixed Point Theorem and Fractional Differential Equations with Multiple Delays Related with Chaos Neuron Models
}

\author{
Toshiharu Kawasaki, Masashi Toyoda \\ Faculty of Engineering, Tamagawa University, Tokyo, Japan \\ Email: toshiharu.kawasaki@nifty.ne.jp, mss-toyoda@eng.tamagawa.ac.jp
}

Received 9 July 2015; accepted 27 November 2015; published 30 November 2015

Copyright (C) 2015 by authors and Scientific Research Publishing Inc.

This work is licensed under the Creative Commons Attribution International License (CC BY).

http://creativecommons.org/licenses/by/4.0/

\section{(c) (i) Open Access}

\section{Abstract}

In this paper, we show a fixed point theorem which deduces to both of Lou's fixed point theorem and de Pascale and de Pascale's fixed point theorem. Moreover, our result can be applied to show the existence and uniqueness of solutions for fractional differential equations with multiple delays. Using the theorem, we discuss the fractional chaos neuron model.

\section{Keywords}

Fixed Point Theorem, Ordinary Differential Equation, Delay Differential Equation, Fractional Differential Equation, Fractional Chaos Neuron Model

\section{Introduction}

The following was the famous fixed point theorem introduced by Banach in 1922.

The Banach contraction principle ([1]). Let $(X, d)$ be a complete metric space, let $F$ be a nonempty closed subset of $X$ and let $A$ be a mapping from $F$ into itself. Suppose that there exist $\beta \in[0,1)$ such that

$$
d(A u, A v) \leq \beta d(u, v)
$$

for any $u, v \in F$. Then A has a unique fixed point in $F$.

In 1999 Lou proved the following fixed point theorem.

Lou's fixed point theorem ([2]). Let $I=[0, T]$, let $\left(E,\|\cdot\|_{E}\right)$ be a Banach space, let $C(I, E)$ be the Banach space consisting of all continuous mappings from I into $E$ with norm

$$
\|u\|=\max \left\{\|u(t)\|_{E} \mid t \in I\right\}
$$


for any $u \in C(I, E)$, let $F$ be a nonempty closed subset of $C(I, E)$ and let $A$ be a mapping from $F$ into itself. Suppose that there exist $\alpha, \beta \in[0,1)$ and $K \in[0, \infty)$ such that

$$
\|A u(t)-A v(t)\|_{E} \leq \beta\|u(t)-v(t)\|_{E}+\frac{K}{t^{\alpha}} \int_{0}^{t}\|u(s)-v(s)\|_{E} \mathrm{~d} s
$$

for any $u, v \in F$ and for any $t \in I \backslash\{0\}$. Then A has a unique fixed point in $F$.

Moreover, in 2002 de Pascale and de Pascale proved the following fixed point theorem.

De Pascale-de Pascale's fixed point theorem $([3])$. Let $I=[1, \infty)$, let $\left(E,\|\cdot\|_{E}\right)$ be a Banach space, let $B C(I, E)$ be the Banach space consisting of all bounded continuous mappings from I into $E$ with norm

$$
\|u\|=\sup \left\{\|u(t)\|_{E} \mid t \in I\right\}
$$

for any $u \in B C(I, E)$, let $F$ be a nonempty closed subset of $B C(I, E)$ and let $A$ be a mapping from $F$ into itself. Suppose that there exist $\alpha \in(1, \infty), \quad \beta \in[0,1)$ and $K \in[0, \infty)$ such that

$$
\|A u(t)-A v(t)\|_{E} \leq \beta\|u(t)-v(t)\|_{E}+\frac{K}{t^{\alpha}} \int_{1}^{t}\|u(s)-v(s)\|_{E} \mathrm{~d} s
$$

for any $u, v \in F$ and for any $t \in I$. Then $A$ has a unique fixed point in $F$.

In this paper, using the Banach contraction principle, we show a fixed point theorem which deduces to both of Lou's fixed point theorem and de Pascale and de Pascale's fixed point theorem. Moreover, our results can be applied to show the existence and uniqueness of solutions for fractional differential equations with multiple delays. Using the theorem, we discuss the fractional chaos neuron model [4].

\section{Fixed Point Theorem}

In this section, we show a fixed point theorem. It deduces to Lou's fixed point theorem [2] and de Pascale and de Pascale's fixed point theorem [3].

Definition 1. Let $I$ be an arbitrary finite or infinite interval, let $J$ be an interval with $I \subset J$, let $\left(E,\|\cdot\|_{E}\right)$ be a Banach space, let $B C(I, E)$ be the Banach space consisting all bounded continuous mappings from $I$ into $E$ with norm

$$
\|u\|=\sup \left\{\|u(t)\|_{E} \mid t \in I\right\}
$$

for any $u \in B C(I, E)$, let $B C(I, E)$ be the Banach space consisting all bounded continuous mappings from $J$ into $E$ with norm

$$
\|u\|_{J}=\sup \left\{\|u(t)\|_{E} \mid t \in J\right\}
$$

for any $u \in B C(I, E)$, let $F$ be a nonempty closed subset of $B C(I, E)$, and let $\phi$ be a mapping from $J \backslash I$ into $E$. Define a mapping $u_{\phi}$ by

$$
u_{\phi}= \begin{cases}u & \text { on } I, \\ \phi & \text { on } J \backslash I\end{cases}
$$

for any $u \in F$. We say $F$ satisfies (*) for $\phi$ if $(*) u_{\phi} \in B C(J, E)$ holds for any $u \in F$.

Theorem 1. Let $I$ be an arbitrary finite or infinite interval, let $J_{0}, J$ be intervals with $I \subset J_{0} \subset J$, let $\left(E,\|\cdot\|_{E}\right)$ be a Banach space, let $B C(I, E)$ be the Banach space consisting all bounded continuous mappings from I into $E$ with norm

$$
\|u\|=\sup \left\{\|u(t)\|_{E} \mid t \in I\right\}
$$

for any $u \in B C(I, E)$, and let $F$ be a nonempty closed subset of $B C(I, E)$. Suppose that there exists a mapping $\phi$ from $J \backslash I$ into $E$ such that $F$ satisfies (*) for $\phi$. Let A be a mapping from $F$ into itself. Suppose that there exist $\beta \in[0,1)$, a mapping $G$ from $I \times J_{0}$ into $[0, \infty)$ integrable with respect to the second variable for any the first variable, mappings $\gamma, \delta$ from $I$ into $J_{0}$ with $\gamma \leq \delta, n \in \mathbb{N}$, and mappings $\eta_{i} \in C\left(J_{0}, J\right)$ for any 
$i=1, \cdots, n$ such that

$\left(H_{1}\right)$ for any $u, v \in F$ and for any $t \in I$

$$
\|A u(t)-A v(t)\|_{E} \leq \beta\|u(t)-v(t)\|_{E}+\int_{\gamma(t)}^{\delta(t)} G(t, s) \sum_{i=1}^{n}\left\|u_{\phi}\left(\eta_{i}(s)\right)-v_{\phi}\left(\eta_{i}(s)\right)\right\|_{E} \mathrm{~d} s
$$

$\left(H_{2}\right)$ there exist $\alpha \in[0, \infty), K \in[0, \infty), m, M \in(0, \infty)$ with $m \leq M$ and $y \in B C(J,[m, M])$ such that

1) $\beta+n K \alpha \in[0,1)$;

2) $y\left(\eta_{i}(t)\right) \leq K y(t)$ for any $t \in J_{0}$ and for any $i=1, \cdots, n$;

3) $\int_{\gamma(t)}^{\delta(t)} G(t, s) y(s) \mathrm{d} s \leq \alpha y(t)$ for any $t \in I$.

Then $A$ has a unique fixed point in $F$.

Proof. By $\left(H_{1}\right)$ we obtain

$$
\|A u(t)-A v(t)\|_{E} \leq \beta\|u(t)-v(t)\|_{E}+\int_{\gamma(t)}^{\delta(t)} G(t, s) \sum_{i=1}^{n}\left\|u_{\phi}\left(\eta_{i}(s)\right)-v_{\phi}\left(\eta_{i}(s)\right)\right\|_{E} \mathrm{~d} s
$$

for any $u, v \in F$ and for any $t \in I$. By $\left(H_{2}\right)$ there exists $y \in B C(J,[m, M])$, that is, $m \leq y(t) \leq M$ for any $t \in J$. Define a new norm $\|\cdot\|_{y}$ in $B C(I, E)$ by

$$
\|u\|_{y}=\sup \left\{\frac{1}{y(t)}\|u(t)\|_{E} \mid t \in I\right\} .
$$

Since

$$
\frac{1}{M}\|u\| \leq\|u\|_{y} \leq \frac{1}{m}\|u\|
$$

$\|\cdot\|_{y}$ is equivalent of $\|\cdot\|$. Define a metric $d$ in $F$ by

$$
d(u, v)=\sup \left\{\frac{1}{y(t)}\left|u_{\phi}(t)-v_{\phi}(t) \|_{E}\right| t \in J\right\} .
$$

Since $u_{\phi}(t)=v_{\phi}(t)=\phi(t)$ for any $t \in J \backslash I$, we obtain

$$
d(u, v)=\sup \left\{\frac{1}{y(t)}\left\|u_{\phi}(t)-v_{\phi}(t)\right\|_{E} \mid t \in J\right\}=\sup \left\{\frac{1}{y(t)}\|u(t)-v(t)\|_{E} \mid t \in J\right\}=\|u-v\|_{y}
$$

and hence $(F, d)$ is a complete metric space. We obtain

$$
\begin{aligned}
& \frac{1}{y(t)}\|A u(t)-A v(t)\|_{E} \\
& \leq \frac{\beta}{y(t)}\|u(t)-v(t)\|_{E}+\frac{1}{y(t)} \int_{\gamma(t)}^{\delta(t)} G(t, s) \sum_{i=1}^{n}\left\|u_{\phi}\left(\eta_{i}(s)\right)-v_{\phi}\left(\eta_{i}(s)\right)\right\|_{E} \mathrm{~d} s \\
& \leq \beta d(u, v)+\frac{1}{y(t)} \int_{\gamma(t)}^{\delta(t)} G(t, s) \sum_{i=1}^{n} d(u, v) y\left(\eta_{i}(s)\right) \mathrm{d} s \\
& \leq \beta d(u, v)+\frac{d(u, v)}{y(t)} \int_{\gamma(t)}^{\delta(t)} G(t, s) \sum_{i=1}^{n} K y(s) \mathrm{d} s \\
& =\beta d(u, v)+\frac{n K d(u, v)}{y(t)} \int_{\gamma(t)}^{\delta(t)} G(t, s) y(s) \mathrm{d} s \\
& \leq(\beta+n K \alpha) d(u, v)
\end{aligned}
$$

for any $u, v \in F$ and for any $t \in I$. Since $(A u)_{\phi}(t)=(A v)_{\phi}(t)=\phi(t)$ for any $t \in J \backslash I$, we obtain 


$$
\begin{aligned}
d(A u, A v) & =\sup \left\{\frac{1}{y(t)}\left\|(A u)_{\phi}(t)-(A v)_{\phi}(t)\right\|_{E} \mid t \in J\right\} \\
& =\sup \left\{\frac{1}{y(t)}\|A u(t)-A v(t)\|_{E} \mid t \in J\right\} \leq(\beta+n K \alpha) d(u, v),
\end{aligned}
$$

that is, $A$ is a contraction mapping. By the Banach contraction principle $A$ has a unique fixed point in $F$.

The following remarks show that our fixed point theorem derives Lou's fixed point theorem [2] and de Pascale and de Pascale's fixed point theorem [3]. The proofs are owed to [5].

Remark 1. By Theorem 1 we can obtain Lou's fixed point theorem [2]. Actually let $I=J_{0}=J=[0, T]$, let $\left(E,\|\cdot\|_{E}\right)$ be a Banach space, let $C(I, E)$ be the Banach space consisting of all continuous mappings from $I$ into $E$ with norm

$$
\|u\|=\max \left\{\|u(t)\|_{E} \mid t \in I\right\}
$$

for any $u \in C(I, E)$, and let $F$ be a nonempty closed subset of $C(I, E)$. F satisfies (*) for the null mapping. Note that, since $I$ is a finite interval, $C(I, E)$ is equivalent to $B C(I, E)$. Let $A$ be a mapping from $F$ into itself. Suppose that there exist $\alpha_{0}, \beta \in[0,1)$ and $K_{0} \in[0, \infty)$ such that

$$
\|A u(t)-A v(t)\|_{E} \leq \beta\|u(t)-v(t)\|_{E}+\frac{K_{0}}{t^{\alpha_{0}}} \int_{0}^{t}\|u(s)-v(s)\|_{E} \mathrm{~d} s
$$

for any $u, v \in F$ and for any $t \in I \backslash\{0\}$. Note that $A$ is continuous. Therefore by the l'Hopital theorem we obtain

$$
\begin{aligned}
\|A u(0)-A v(0)\|_{E} & \leq \beta\|u(0)-v(0)\|_{E}+\lim _{t \rightarrow+0} \frac{K_{0}}{t^{\alpha_{0}}} \int_{0}^{t}\|u(s)-v(s)\|_{E} \mathrm{~d} s \\
& =\beta\|u(0)-v(0)\|_{E}+\lim _{t \rightarrow+0} \frac{K_{0}}{\alpha_{0} t^{\alpha_{0}-1}}\|u(t)-v(t)\|_{E} \\
& =\beta\|u(0)-v(0)\|_{E}
\end{aligned}
$$

for any $u, v \in F$. Put

$$
G(t, s)= \begin{cases}\frac{K_{0}}{t_{0}} & \text { if } 0<t \leq T, \\ 0 & \text { if } t=0,\end{cases}
$$

$\gamma(t)=0, \delta(t)=t, \quad n=1$ and $\eta_{1}(t)=t$. Then we obtain

$$
\begin{aligned}
& \|A u(t)-A v(t)\|_{E} \\
& \leq \begin{cases}\beta\|u(t)-v(t)\|_{E}+\frac{K_{0}}{t^{\alpha_{0}}} \int_{0}^{t}\|u(s)-v(s)\|_{E} \mathrm{~d} s & \text { if } 0<t \leq T, \\
\beta\|u(0)-v(0)\|_{E} & \text { if } t=0\end{cases} \\
& =\beta\|u(t)-v(t)\|_{E}+\int_{\gamma(t)}^{\delta(t)} G(t, s) \sum_{i=1}^{n}\left\|u\left(\eta_{i}(s)\right)-v\left(\eta_{i}(s)\right)\right\|_{E} \mathrm{~d} s
\end{aligned}
$$

for any $u, v \in F$ and for any $t \in I$, that is, $\left(H_{1}\right)$ holds. Take $\tau \in(0, \infty)$ satisfying $K_{0} \tau^{1-\alpha_{0}}<1-\beta$. Put $\alpha=K_{0} \tau^{1-\alpha_{0}}, \quad K=1, \quad m=1, \quad M=\mathrm{e}^{\frac{T}{\tau}-1}$ and

$$
y(t)= \begin{cases}1 & \text { if } 0 \leq t \leq \tau, \\ \mathrm{e}^{\frac{t}{\tau}-1} & \text { if } \tau \leq t \leq T .\end{cases}
$$

Then (1) and (2) of $\left(H_{2}\right)$ hold. Moreover, if $0 \leq t \leq \tau$, then 


$$
\int_{\gamma(t)}^{\delta(t)} G(t, s) y(s) \mathrm{d} s=\int_{0}^{t} \frac{K_{0}}{t^{\alpha_{0}}} \mathrm{~d} s=K_{0} t^{1-\alpha_{0}}=\alpha y(t)
$$

if $\tau \leq t \leq T$, then

$$
\int_{\gamma(t)}^{\delta(t)} G(t, s) y(s) \mathrm{d} s=\int_{0}^{\tau} \frac{K_{0}}{t^{\alpha_{0}}} \mathrm{~d} s+\int_{\tau}^{t} \frac{K_{0}}{t^{\alpha_{0}}} \mathrm{e}^{\frac{s}{\tau}-1} \mathrm{~d} s=\frac{K_{0} \tau}{t^{\alpha_{0}}} \mathrm{e}^{\frac{t}{\tau}-1} \leq \alpha y(t),
$$

that is, (3) of $\left(\mathrm{H}_{2}\right)$ holds. Therefore, by Theorem $1 \mathrm{~A}$ has a unique fixed point in $F$.

Remark 2. By Theorem 1 we can obtain de Pascale and de Pascale's fixed point theorem [3]. Actually let $I=J_{0}=J=[1, \infty)$, let $\left(E,\|\cdot\|_{E}\right)$ be a Banach space, let $B C(I, E)$ be the Banach space consisting of all bounded continuous mappings from $I$ into $E$ with norm

$$
\|u\|=\sup \left\{\|u(t)\|_{E} \mid t \in I\right\}
$$

for any $u \in B C(I, E)$, and let $F$ be a nonempty closed subset of $B C(I, E)$. F satisfies (*) for the null mapping. Let $A$ be a mapping from $F$ into itself. Suppose that there exist $\alpha_{0} \in(1, \infty), \beta \in[0,1)$ and $K_{0} \in[0, \infty)$ such that

$$
\|A u(t)-A v(t)\|_{E} \leq \beta\|u(t)-v(t)\|_{E}+\frac{K_{0}}{t^{\alpha_{0}}} \int_{1}^{t}\|u(s)-v(s)\|_{E} \mathrm{~d} s
$$

for any $u, v \in F$ and for any $t \in I$. Put

$$
G(t, s)=\frac{K_{0}}{t^{\alpha_{0}}},
$$

$\gamma(t)=1, \delta(t)=t, n=1$ and $\eta_{1}(t)=t$. Then we obtain

$$
\begin{aligned}
\|A u(t)-A v(t)\|_{E} & \leq \beta\|u(t)-v(t)\|_{E}+\frac{K_{0}}{t^{\alpha_{0}}} \int_{1}^{t}\|u(s)-v(s)\|_{E} \mathrm{~d} s \\
& =\beta\|u(t)-v(t)\|_{E}+\int_{\gamma(t)}^{\delta(t)} G(t, s) \sum_{i=1}^{n}\left\|u\left(\eta_{i}(s)\right)-v\left(\eta_{i}(s)\right)\right\|_{E} \mathrm{~d} s
\end{aligned}
$$

for any $u, v \in F$ and for any $t \in I$, that is, $\left(H_{1}\right)$ holds. Take $c \in(0, \infty)$ and $\tau \in(1, \infty)$ satisfying $K_{0}\left(c^{-1}+\tau^{1-\alpha_{0}}\right)<1-\beta$. Put $\alpha=K_{0}\left(c^{-1}+\tau^{1-\alpha_{0}}\right), \quad K=1, \quad m=\mathrm{e}^{c}, \quad M=\mathrm{e}^{c \tau}$ and

$$
y(t)= \begin{cases}\mathrm{e}^{c t} & \text { if } 1 \leq t \leq \tau, \\ \mathrm{e}^{c \tau} & \text { if } \tau \leq t .\end{cases}
$$

Then (1) and (2) of $\left(H_{2}\right)$ hold. Moreover, if $1 \leq t \leq \tau$, then

$$
\int_{\gamma(t)}^{\delta(t)} G(t, s) y(s) \mathrm{d} s=\int_{1}^{t} \frac{K_{0}}{t^{\alpha_{0}}} \mathrm{e}^{c s} \mathrm{~d} s=\frac{K_{0}}{c t^{\alpha_{0}}}\left(\mathrm{e}^{c t}-\mathrm{e}^{c}\right) \leq K_{0} c^{-1} \mathrm{e}^{c t} \leq \alpha y(t)
$$

if $\tau \leq t$, then

$$
\begin{aligned}
\int_{\gamma(t)}^{\delta(t)} G(t, s) y(s) \mathrm{d} s & =\int_{1}^{\tau} \frac{K_{0}}{t^{\alpha_{0}}} \mathrm{e}^{c s} \mathrm{~d} s+\int_{\tau}^{t} \frac{K_{0}}{t^{\alpha_{0}}} \mathrm{e}^{c \tau} \mathrm{d} s=\frac{K_{0}}{c t^{\alpha_{0}}}\left(\mathrm{e}^{c \tau}-\mathrm{e}^{c}\right)+\frac{K_{0} \mathrm{e}^{c \tau}}{t^{\alpha_{0}}}(t-\tau) \\
& \leq K_{0}\left(c^{-1}+\tau^{1-\alpha_{0}}\right) \mathrm{e}^{c \tau}=\alpha y(t),
\end{aligned}
$$

that is, (3) of $\left(H_{2}\right)$ holds. Therefore, by Theorem $1 A$ has a unique fixed point in $F$.

\section{Fractional Differential Equations with Multiple Delays}

In this section, by using Theorem 1, we show the existence and uniqueness of solutions for fractional differential equations with multiple delays. Throughout this paper, the fractional derivative means the Caputo-Riesz derivative ${ }^{c} D^{\eta}$ defined by

$$
{ }^{c} D^{\eta} u(t)=\frac{1}{\Gamma(m-\eta)} \int_{0}^{t}(t-s)^{m-\eta-1} \frac{\mathrm{d}^{m}}{\mathrm{~d} s^{m}} u(s) \mathrm{d} s
$$


for any $\eta \in(0, \infty)$ and for any function $u$, where $\Gamma$ is the gamma function and $m$ is a natural number with $m-1 \leq \eta<m$; for instance, see [6].

Theorem 2. Let $\left(E,\|\cdot\|_{E}\right)$ be a Banach space, let $C\left([0, T] \times E^{n}, E\right)$ be the space consisting of all continuous mappings from $[0, T] \times E^{n}$ into $E$ and let $f \in C\left([0, T] \times E^{n}, E\right)$ satisfying

$\left(H_{f}\right)$ there exist $L_{i} \in[0, T](i=1, \cdots, n)$ such that

$$
\left\|f\left(t, x_{1}, \cdots, x_{n}\right)-f\left(t, y_{1}, \cdots, y_{n}\right)\right\|_{E} \leq \sum_{i=1}^{n} L_{i}\left\|x_{i}-y_{i}\right\|_{E}
$$

for any $t \in[0, T]$ and for any $x_{i}, y_{i} \in E$.

Let $C([0, T], E)$ be the Banach space consisting of all continuous mappings from $[0, T]$ into $E$, let $C([0, T],[0, \infty))$ be the space consisting of all continuous mappings from $[0, T]$ into $[0, \infty)$ and let $C((-\infty, 0], E)$ be the space consisting of all continuous mappings from $(-\infty, 0]$ into $E$. Then the following fractional differential equation with multiple delays

$$
{ }^{c} D^{\eta} u(t)=f\left(t, u(t), u_{\phi}\left(t-\tau_{2}(t)\right), \cdots, u_{\phi}\left(t-\tau_{n}(t)\right)\right) \quad(t \in[0, T]),
$$

where $\eta \in(0,1],{ }^{c} D^{\eta}$ is the $\eta$-order Caputo-Riesz derivative, $\tau_{i} \in C([0, T],[0, \infty))$ and $\phi \in C((-\infty, 0], E)$, have a unique solution in $\{u \mid u \in C([0, T], E)$ and $u(0)=\phi(0)\}$.

Proof. Put $I=J_{0}=[0, T], \quad \tau=\inf \left\{t-\tau_{i}(t) \mid t \in I, i=2, \cdots, n\right\}, \quad J=[\tau, T]$ and

$$
F=\{u \mid u \in C(I, E) \text { and } u(0)=\phi(0)\} \text {. }
$$

Then $F$ is closed. Since $\phi \in C([\tau, 0), E)$ and $u(0)=\phi(0)$ for any $u \in F$, we obtain $u_{\phi} \in C(J, E)$ for any $u \in F$. Therefore, $F$ satisfies $(*)$ for $\phi$. By direct computations, $u \in C(I, E)$ is a solution of the equation above if and only if it is a solution of the following integral equation:

$$
u(t)=\phi(0)+\frac{1}{\Gamma(\eta)} \int_{0}^{t}(t-s)^{\eta-1} f\left(s, u(s), u_{\phi}\left(s-\tau_{2}(s)\right), \cdots, u_{\phi}\left(s-\tau_{n}(s)\right)\right) \mathrm{d} s .
$$

Define a mapping $A$ by

$$
A u(t)=\phi(0)+\frac{1}{\Gamma(\eta)} \int_{0}^{t}(t-s)^{\eta-1} f\left(s, u(s), u_{\phi}\left(s-\tau_{2}(s)\right), \cdots, u_{\phi}\left(s-\tau_{n}(s)\right)\right) \mathrm{d} s
$$

for any $u \in F$. Since $A u(0)=\phi(0)$, we obtain $A u \in F$. We show that $A$ has a unique fixed point. Indeed, we obtain

$$
\begin{aligned}
\|A u(t)-A v(t)\|_{E} & \leq \frac{1}{\Gamma(\eta)} \int_{0}^{t}(t-s)^{\eta-1} \sum_{i=1}^{n} L_{i}\left\|u_{\phi}\left(s-\tau_{i}(s)\right)-v_{\phi}\left(s-\tau_{i}(s)\right)\right\|_{E} \\
& \leq \frac{L}{\Gamma(\eta)} \int_{0}^{t}(t-s)^{\eta-1} \sum_{i=1}^{n}\left\|u_{\phi}\left(\eta_{i}(s)\right)-v_{\phi}\left(\eta_{i}(s)\right)\right\|_{E} \mathrm{~d} s,
\end{aligned}
$$

where $\tau_{1}(t)=0, L=\max \left\{L_{1}, \cdots, L_{n}\right\}$ and $\eta_{i}(t)=t-\tau_{i}(t)(i=1, \cdots, n)$. Put $\beta=0$,

$$
G(t, s)= \begin{cases}\frac{L}{\Gamma(\eta)}(t-s)^{\eta-1} & \text { if } 0 \leq s<t, \\ 0 & \text { if } t \leq s,\end{cases}
$$

$\gamma(t)=0$ and $\delta(t)=t$. Then $\left(H_{1}\right)$ holds. Take $\alpha$ with $0<n \alpha<1$ and take $c$ with $c^{\eta} \geq \frac{L}{\alpha}$. Put $K=1$, $m=\mathrm{e}^{c \tau}, \quad M=\mathrm{e}^{c T}$ and $y(t)=\mathrm{e}^{c t}$. Then (1) and (2) of $\left(H_{2}\right)$ hold. Moreover, since

$$
\int_{\gamma(t)}^{\delta(t)} G(t, s) y(s) \mathrm{d} s=\frac{L}{\Gamma(\eta)} \int_{0}^{t}(t-s)^{\eta-1} \mathrm{e}^{c s} \mathrm{~d} s=\frac{L \mathrm{e}^{c t}}{c^{\eta} \Gamma(\eta)} \int_{0}^{c t} s^{\eta-1} \mathrm{e}^{-s} \mathrm{~d} s \leq \frac{L}{c^{\eta}} \mathrm{e}^{c t} \leq \alpha y(t),
$$


(3) of $\left(H_{2}\right)$ holds. Therefore, by Theorem $1 A$ has a unique fixed point in $F$.

By using Theorem 2, we discuss the fractional chaos neuron model [4].

Example 1. We consider the following fractional differential equation with delay

$$
{ }^{c} D^{\eta} u(t)=-\beta u(t)+\sin \frac{\pi u_{\phi}(t-\tau)}{2 T_{0}} \quad(t \in[0, T]),
$$

where $\eta \in(0,1], \quad \beta, \tau \in[0, \infty), T_{0} \in(0, \infty)$ and $\phi \in C([-\tau, 0], \mathbb{R})$. In this equation, $u(t)$ is an internal state of the neuron at time $t, \beta$ is a dissipative parameter and $\tau$ is delay time. Moreover, we use a sinusoidal function with a periodic parameter $T_{0}$ as an activation to be related to the output of the neuron. This equation is called the fractional chaos neuron model [4]. Put $E=\mathbb{R}, n=2, \tau_{2}(t)=\tau$ and $f\left(t, x_{1}, x_{2}\right)=-\beta x_{1}+\sin \frac{\pi x_{2}}{2 T_{0}}$. Since

$$
\left|f\left(t, x_{1}, x_{2}\right)-f\left(t, y_{1}, y_{2}\right)\right| \leq|\beta|\left|x_{1}-y_{1}\right|+\left|\sin \frac{\pi x_{2}}{2 T_{0}}-\sin \frac{\pi y_{2}}{2 T_{0}}\right| \leq|\beta|\left|x_{1}-y_{1}\right|+\frac{\pi}{2 T_{0}}\left|x_{2}-y_{2}\right|,
$$

$f$ satisfies $\left(H_{f}\right)$ for $L_{1}=|\beta|$ and $L_{2}=\frac{\pi}{2 T_{0}}$. Therefore, by Theorem 2 the equation above has a unique solution in $\{u \mid u \in C([0, T], \mathbb{R})$ and $u(0)=\phi(0)\}$. For analysis of neural networks using fixed point theorems, see [7] [8].

\section{Acknowledgements}

The authors would like to thank the referee for valuable comments.

\section{References}

[1] Banach, S. (1922) Sur les opérations dans les ensembles abstraits et leur application aux équations integrals. Fundamenta Mathematicae, 3, 133-181.

[2] Lou, B. (1999) Fixed Points for Operators in a Space of Continuous Functions and Applications. Proceedings of the American Mathematical Society, 127, 1159-2264. http://dx.doi.org/10.1090/S0002-9939-99-05211-9

[3] de Pascale, E. and de Pascale, L. (2002) Fixed Points for Some Non-Obviously Contractive Operators. Proceedings of the American Mathematical Society, 130, 3249-3254. http://dx.doi.org/10.1090/S0002-9939-02-06704-7

[4] Matsuzaki, T. and Nakagawa, M. (2003) A Chaos Neuron Model with Fractional Differential Equation. Journal of the Physical Society of Japan, 72, 2678-2684. http://dx.doi.org/10.1143/JPSJ.72.2678

[5] Suzuki, T. (2006) Lou's Fixed Point Theorem in a Space of Continuous Mappings. Journal of the Mathematical Society of Japan, 58, 769-774. http://dx.doi.org/10.2969/jmsj/1156342037

[6] Kilbas, A.A., Srivastava, H.M. and Trujillo, J.J. (2006) Theory and Applications of Fractional Differential Equations. Elsevier, Amsterdam.

[7] Lai, X. and Zhang, Y. (2012) Fixed Point and Asymptotic Analysis of Cellular Neural Networks. Journal of Applied Mathematics, 2012, Article ID: 689845.

[8] Zhang, Y. and Luo, Q. (2013) Global Exponential Stability of Impulsive Cellular Neural Networks with Time-Varying Delays via Fixed Point Theory. Advances in Difference Equations, 2013, 2013:23. 